\title{
A Block-Based Arithmetic Entropy Encoding Scheme for Medical Images
}

\author{
Urvashi Sharma, Jaypee University of Information Technology, India \\ Meenakshi Sood, National Institute of Technical Teachers Training and Research, India \\ Emjee Puthooran, Jaypee University of Information Technology, India \\ Yugal Kumar, Jaypee University of Information Technology, India
}

\begin{abstract}
The digitization of human body, especially for treatment of diseases can generate a large volume of data. This generated medical data has a large resolution and bit depth. In the field of medical diagnosis, lossless compression techniques are widely adopted for the efficient archiving and transmission of medical images. This article presents an efficient coding solution based on a predictive coding technique. The proposed technique consists of Resolution Independent Gradient Edge Predictor16 (RIGED16) and Block Based Arithmetic Encoding (BAAE). The objective of this technique is to find universal threshold values for prediction and provide an optimum block size for encoding. The validity of the proposed technique is tested on some real images as well as standard images. The simulation results of the proposed technique are compared with some well-known and existing compression techniques. It is revealed that proposed technique gives a higher coding efficiency rate compared to other techniques.
\end{abstract}

\section{KEYWORDS}

Lossless Compression, Gradient Edge Detection, Resolution Independent Gradient Edge, Data Compression

\section{INTRODUCTION}

In current health care practices, standard medical imaging systems are used for medical diagnosis. With the advancement in digital and scanning technologies, these medical imaging systems have become an important part of the diagnostic systems. These systems produce accurate images of high quality with high resolution and bit depths. Such improvement in imaging systems produces large amount of medical data to be processed, archived and transmitted. During past few decades, enormous amount of digital imaging data was generated, especially in biomedical field. The volumetric scanning technologies, such as Computed Tomography (CT) and Magnetic Resonance Imaging (MRI) generate large number of image frames and require huge amount of space for storage. These image frames consume more bandwidth for transmission. It is a complex task to handle transmission, archiving and manage the data produced during radiological process (Ravishankar \& Bresler, 2011; Bhardwaj, 2017). These facts motivate the research in the area of efficient compression techniques for high resolution and higher bit depth images. The aim of image compression is to remove redundant or irrelevant data from the image such that it could be stored, transmit and processed effectively (Williams, 1991; Bell et al., 1990). Further, the compression techniques are broadly divided into two categories i.e. lossless and lossy. But in the field of medical diagnosis, lossless techniques are widely adopted because the

\section{DOI: 10.4018/IJHISI.2020070104}

This article, originally published under IGI Global's copyright on March 13, 2020 will proceed with publication as an Open Access article starting on January 14, 2021 in the gold Open Access journal, International Journal of Healthcare Information Systems and Informatics (converted to gold Open Access January 1, 2021), and will be distributed under the terms of the Creative Commons Attribution License (http:// creativecommons.org/licenses/by/4.0/) which permits unrestricted use, distribution, and production in any medium, provided the author of the original work and original publication source are properly credited. 
data is not lost during recovery process. On the other hand, lossy technique doesn't provide accurate recovery at the receiver side and may lead to wrong diagnosis (Al-Khafaji, 2013; Al-Khafaji \& Ghanim, 2017; Kabir \& Mondal, 2018).

In literature, different compression techniques have been reported for medical diagnosis such as dictionary encoding, transformation encoding and predictive encoding techniques. It is noted the transform-based standards cannot provide higher compression rate (Al-Khafaji \& Ghanim, 2017). These standards contain Discrete Cosine Transform (DCT) and Discrete Wavelet Transform (DWT) for compression. Other side, predictive based compression techniques perform well and provide higher compression rate with low complexity (Gupta et al., 2013). The joint photographic experts group- lossless (JPEG-LS) (DIS, 1991; Pennebaker \& Mitchell, 1992; Weinberger et al., 2000) and context-based, adaptive, lossless image coding (CALIC) are standard predictive coding techniques for lossless compression of medical images (Wu \& Memon, 1997). The JPEG-LS is based on low complexity lossless compression (LOCO-I) algorithm using standard median edge detector (MED) and golomb code (Weinberger et al., 2000; Matsuda et al., 2000). While, CALIC technique consists of two mechanisms, one is used for prediction and other is applied for image encoding. The prediction is done through gradient adjust predictor (GAP) and image encoding is performed by using arithmetic encoding. It is noticed that GAP predictor is more efficient than MED, but, is computationally extensive (Avramovic \& Savic, 2011). So, the CALIC is more efficient than JPEG-LS in terms of Bits per Pixel (BPP). Many researchers have worked on predictive coding techniques and adopted lossless compression for medical images.

Many researchers have worked on predictive coding techniques and adopted lossless compression for medical images. Avramovic and Savic developed a predictive algorithm based on edge detection and local gradients (Avramovic and Savic, 2011). In this work, the strengths of 2D standard predictors are analyzed. The analysis showed that the GAP predictor performs well for medical images. AlMahmood \& Al-Rubaye adopted a compression method that is based on a combination between predictive coding and bit plane slicing for compression of medical and natural image samples (AlMahmood \& Al-Rubaye, 2014). This compression technique discards the lowest order bits and exploits only higher order bits in which most significant bit used predictive coding. Anitha proposed a hybrid technique that combines integer wavelet transforms (IWT) and predictive coding technique to improve the performance of lossless compression (Anitha, 2015). IWT is applied to input image samples and predictive coding was applied to the output of IWT. Entropy and compression ratio are used to evaluate the compression performance. Gupta et al. highlighted the prediction-based compression and combined this predictive coding with IWT for achieving better compression ratio (Gupta et al., 2013). Performance results of proposed technique in terms of Bits per Pixel (BPP) and Peak Signal to Noise Ratio (PSNR) is better achieved by proposed technique as compare to existing JPEG 2000.

In current scenario, advanced scanning techniques (MRI and CT) are applied for medical diagnosis. These techniques have higher bit depth images with improved image quality. This research work presents an efficient coding solution based on predictive coding technique for lossless compression of higher bit depth volumetric medical images. The proposed approach is the combination of Resolution Independent Gradient Edge Detector16 (RIGED16) and the Block Adaptive Arithmetic Encoding (BAAE). The proposed algorithm selects an optimum universal threshold for prediction and an optimal block size of residual image for encoding purpose. The experimental results show that the proposed algorithm achieves higher compression performance as compared to standard lossless compression approaches. Rest of the paper is arranged as follows. Section 2 describes the dataset explored for this work and general overview of predictive coding techniques. In section 3, RIGED16 and BAAE are proposed for lossless compression of 3D sets of medical images of different modalities and resolutions. Section 4 presents different performance parameters used for evaluation of proposed technique. The experimental results are demonstrated and discussed in section 5 . The conclusion of this work is given in section 6 . 


\section{MATERIALS AND METHODS}

\subsection{Dataset}

A set of medical images (MRI and CT-scan) are collected from three different sources for testing and comparative analysis of the proposed algorithm. All collected images are of 16 bit depth. The MRI and CT images are usually 12 to 16 bits deep to cover the maximum depth, but researchers have utilized all 16 bit deep images. The lossless compression algorithm for MR and CT is designed to work with data that is natively of 16 bit depth. Test set I is a standard database contains 16 bit images of different image modalities (CT or MR) and resolutions of $256 \times 256$ and $512 \times 512$ presented in Table 1. The medical images presented in Table 1 belong to Cancer Imaging Archive (The Cancer Imaging Archive, 2017; Clark et al, 2013). Table 2 presents Test set-II. This test set is collected from different local hospitals including MR and CT images of different resolutions. Image slice-1 of volumetric MRI_1 and CT set collected from hospitals are also depicted (Figure 1).

\subsection{General Overview of Predictive Coding Technique}

The correlation between adjacent pixels in 2D image is a measure of spatial (interpixel) redundancy. While, correlation of pixels in adjacent image slices of 3D image is temporal (interframe) redundancy. In predictive compression technique, spatial redundancy is removed by $2 \mathrm{D}$ predictors and statistical redundancy is removed by entropy encoders (Al-Khafaji \& Al-Mahmood, 2016). Prediction and entropy encoding are two major steps of predictive coding for lossless compression of an image (Shridevi \& Vijaykumar, 2012) as shown in Figure 2.

In predictive coding technique, every pixel of an image is predicted individually from the context in raster scan format. After prediction, residual image is obtained by taking difference of original image and predicted image as shown in Figure 2. Residual image has less entropy; hence fewer numbers of bits are used to encode the residual image. Entropy encoder is applied to encode residual image to compress image losslessly. Efficiency of predictor depends on how well it predicts the image

Table 1. Test Set-I: standard dataset composed of CT and MR images of different resolutions

\begin{tabular}{|l|c|c|c|}
\hline Sequence & Resolution & Slices & Bits \\
\hline CT_Lung_R13 (Grove et al, 2015) & $512 \times 512$ & 67 & 17563648 \\
\hline CT_Lung_R4 (Grove et al, 2015) & $512 \times 512$ & 68 & 17825792 \\
\hline MR_Neuro (Barboriak, 2015) & $256 \times 256$ & 176 & 11534336 \\
\hline MR_Breast (Meyer, 2015) & $288 \times 288$ & 60 & 3932160 \\
\hline
\end{tabular}

Table 2. Test Set-II: collected from local hospitals composed of CT and MR images of different resolutions

\begin{tabular}{|l|c|c|c|}
\hline Sequence & Resolution & Slices & Bits \\
\hline MR_1 & $512 \times 512$ & 12 & 3145728 \\
\hline MR_2 & $512 \times 512$ & 20 & 5242880 \\
\hline MR_3 & $256 \times 512$ & 11 & 720896 \\
\hline MR_4 & $512 \times 512$ & 20 & 5242880 \\
\hline MR_5 & $512 \times 512$ & 20 & 5242880 \\
\hline MR_6 & $256 \times 256$ & 40 & 2621440 \\
\hline MR_7 & $256 \times 256$ & 20 & 1310720 \\
\hline CT & $512 \times 512$ & 63 & 16515072 \\
\hline
\end{tabular}


Figure 1. a) $1^{\text {st }}$ slice of MRI_1; b) $1^{\text {st }}$ slice of CT

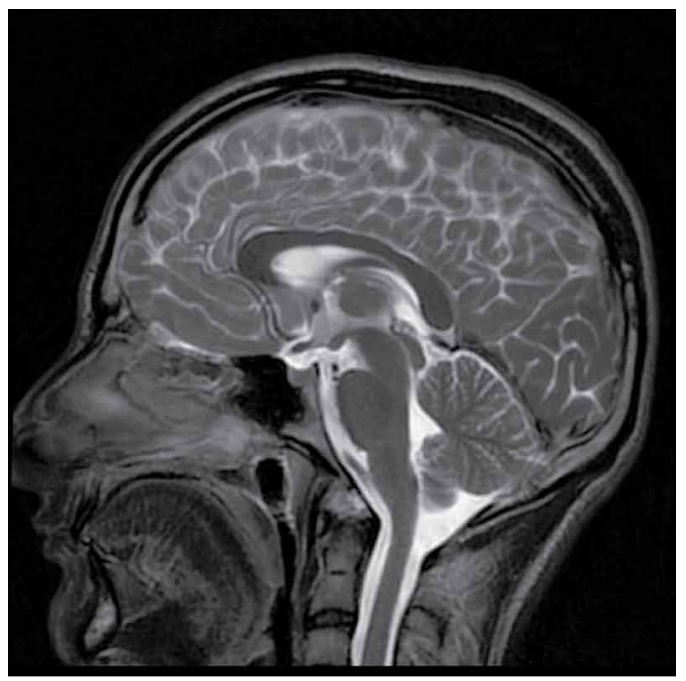

(a)

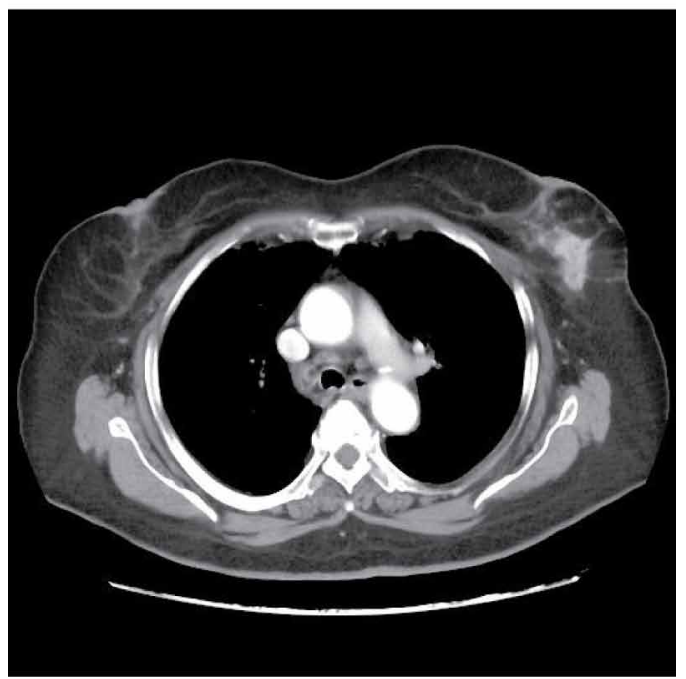

(b)

resulting in reduced entropy of residual image, lesser the entropy better will be the predictor. Entropy encoder's efficiency depends upon its ability to reduce code length.

\subsubsection{Predictors}

$2 \mathrm{D}$ predictor removes the interpixel redundancy from the $2 \mathrm{D}$ images and also for $3 \mathrm{D}$ compression process of volumetric image, operating in frame by frame basis. Common scheme for labeling of causal neighbors in $2 \mathrm{D}$ predictors is shown in Figure 3.

Pixels in causal template are denoted as: 
Figure 2. Basic diagram of predictive coding technique

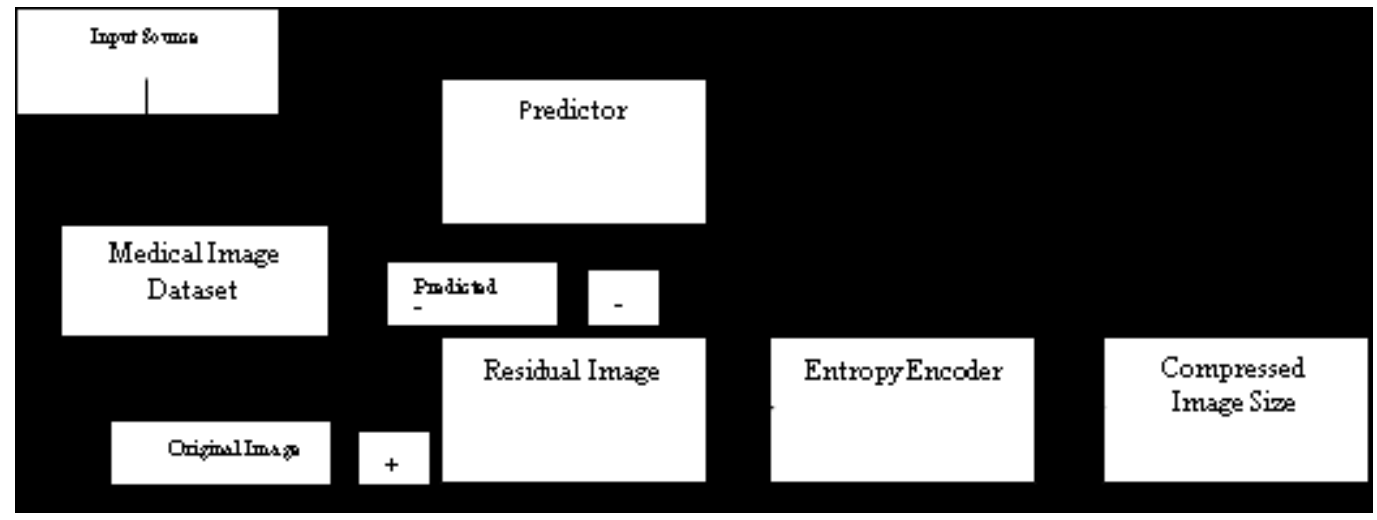

Figure 3. Common scheme of causal template for labeling neighbors

\begin{tabular}{|c|c|c|c|l|}
\hline & & NN & NNE & \\
\hline & NW & N & NE & \\
\hline WW & W & $\mathbf{x}_{\mathbf{i}, \mathrm{j}}$ & & \\
\hline & & & & \\
\hline
\end{tabular}

$$
\left(\begin{array}{l}
X_{\mathrm{i}, \mathrm{j}}=\text { Current pixel to be predicted } \\
X_{N}=X[i, j-1], X_{W}=X[i-1, j] \\
X_{N W}=X[i-1, j-1], X_{N E}=X[i+1, j-1] \\
X_{N N}=X[i, j-2], X_{N W}=X[i-2, j], X_{N N E}=X[i+1, j-2]
\end{array}\right)
$$

MED and GAP are two benchmark algorithms used to minimize the entropy of images. JPEG-LS consists MED predictor which selects the median value among neighboring pixels $\mathrm{N}, \mathrm{W}$ and $\mathrm{W}+\mathrm{N}-$ NW is simple to implement. GAP is based on the gradient estimation and form a part of CALIC. GAP is fixed threshold-based predictor and adapts itself to the intensity gradients of immediate neighbors of predicted pixel. The GED predictor is the combination of MED and GAP and takes the advantage of both the standard predictors. It uses local gradient estimation based on threshold (T) value as that of GAP and like MED, it selects median value between neighboring pixels (Shridevi \& Vijaykumar, 2012). It is also threshold based that is user defined (Avramovic \& Savicl, 2011).

The entropy encoder is a predictive coding technique that converts the residual image into a bit stream of low entropy. The number of bits required for encoding the information depends on entropy. The smaller value of entropy leads to less numbers of bits required for encoding and in turn get higher compression ratio. The different encoding schemes like Huffman, Run-length, Dictionary, Arithmetic and Bit-plane coding are also presented in literature (Anitha, 2015). 


\section{PROPOSED METHODOLOGY}

\subsection{Block Adaptive Arithmetic Encoding (BAAE) Employing RIGED16 Predictor}

The $2 \mathrm{D}$ predictor contains a user defined threshold which makes its simple and efficient. The prediction of an image through GED predictor is based on threshold value. The choice of optimal threshold is very important for efficient prediction. In literature, no specific value for threshold is defined; the different values are used as threshold. This work focuses on the selection of an optimal threshold value that doesn't depend upon the nature of image. Moreover, it is applicable for all modality and resolution of medical images. The RIGED16 is an extension of GED predictor and it is designed to make existing GED resolution and modality independent. The RIGED16 specifies the optimal threshold value for prediction of 16 bit depth images with minimum entropy for residual image. The threshold level for higher bit depth medical images can be up to $2^{16}$. The algorithm of this model is given as:

$\mathrm{Z}_{\mathrm{v}}=|\mathrm{NW}-\mathrm{W}|+|\mathrm{NN}-\mathrm{N}|$

$\mathrm{Z}_{\mathrm{H}}=|\mathrm{WW}-\mathrm{W}|+|\mathrm{NW}-\mathrm{N}|$

if $\mathrm{Z}_{\mathrm{V}}-\mathrm{Z}_{\mathrm{H}}>T$, then $\mathrm{P}_{\mathrm{X}}=\mathrm{W}$

else if $\mathrm{Z}_{\mathrm{V}}-\mathrm{Z}_{\mathrm{H}}<-\mathrm{T}, \mathrm{P}_{\mathrm{X}}=\mathrm{N}$

else $\mathrm{P}_{\mathrm{X}}=\mathrm{N}+\mathrm{W}-\mathrm{NW}$

where $\mathrm{T}=$ Threshold and $\mathrm{Z}_{\mathrm{V}}$ and $\mathrm{Z}_{\mathrm{H}}$ are Vertical and Horizontal Gradients

$\mathrm{T}=768$ (Common threshold for every modality and resolution of medical image)

$\mathrm{T}=2^{9}(512)$ specifically for resolution $256 \times 256$

$\mathrm{T}=2^{10}(1024)$ specifically for resolution $512 \times 512$

After RIGED16 prediction, the residual images are divided into different blocks of varying sizes and having error probabilities in different image regions. The whole image is divided into block size ranging from $4 \times 4$ to $128 \times 128$. On the basis of average absolute error, blocks are grouped and encoded separately through arithmetic encoder. Further, the weighted average of BPP is calculated for every block size. The block size with minimum BPP value is selected. To decompress the images, overhead information is computed which gives the side information of blocks. This information is also considered for BPP calculation of encoded residual image. The block diagram of proposed approach is shown in Figure 4. The working of proposed approach is as follows. An image slice is taken from the image data set and the image resolution is identified. The pixel prediction is done in raster scan order from causal template of an image. The RIGED16 is applied to the image slice that produces predicted image $\mathrm{Z}(\mathrm{x}, \mathrm{y})$. After prediction, residual image or a prediction error image $\mathrm{E}$ $(\mathrm{x}, \mathrm{y})$ is obtained by subtracting $\mathrm{Z}(\mathrm{x}, \mathrm{y})$ from original image $\mathrm{Y}(\mathrm{x}, \mathrm{y})$. The residual image is further divided into optimal size blocks and blocks are grouped on the basis of the average absolute error. Each segmented group of residual image is entropy encoded and it is done through arithmetic encoder. To achieve the lossless compression of images, the abovementioned procedure is repeated for each frame of image and resulted in efficient compression in terms of BPP. 


\section{PERFORMANCE PARAMETERS}

The efficiency of pixel value predictor is inversely related to the entropy of the prediction error image. The entropy describes the number of bits used to represent the information of an image (Shridevi \& Vijaykumar, 2012). The number of slices in image sequence is taken into consideration to calculate the weighted average of entropy. The entropy of an image and weighted average of entropy for complete data-set is calculated as:

$$
H(X)=-\sum_{x \varepsilon Y} p(x) \log p(x)
$$

Entropy weighted Average $=\sum_{N=1}^{M} \frac{m \times n \times S[H(X)]}{m \times n \times S}$

Where, $\mathrm{p}(\mathrm{x})$ is probability of a symbol $\mathrm{X}$.

$\mathrm{M}=$ Total number of datasets to be tested.

$\mathrm{m}, \mathrm{n}=$ Image resolution.

$\mathrm{S}=$ Number of image slices in each data sequence.

The data size of compressed image depends on the BPP along with resolution of an image. Compression Ratio (CR) and BPP are inversely related to each other (Avudaiappan et al., 2017; Puthooran et al., 2013).

$\mathrm{BPP}=\frac{\text { Bit depth of image }}{C R}$

BPP weighted Average $=\sum_{N=1}^{M} \frac{m \times n \times S[B P P]}{m \times n \times S}$

\section{RESULTS AND DISCUSSION}

This section demonstrates simulation results of proposed approach using two test set problems. The performance of the proposed technique is evaluated using image datasets Test Set- 1 and Test Set- 2 . The description of these image datasets is given in Tables 1-2. MATLAB simulation environment is used to implement the proposed approach. The proposed algorithm is also work with the same performance for 12 bit deep images. The simulation results of proposed approach are compared with CALIC, JPEG-LS and other lossless compression techniques (Lucas et al., 2017; 3D-Calic implementation, 2017; JPEG 2000 codec, 2017). 


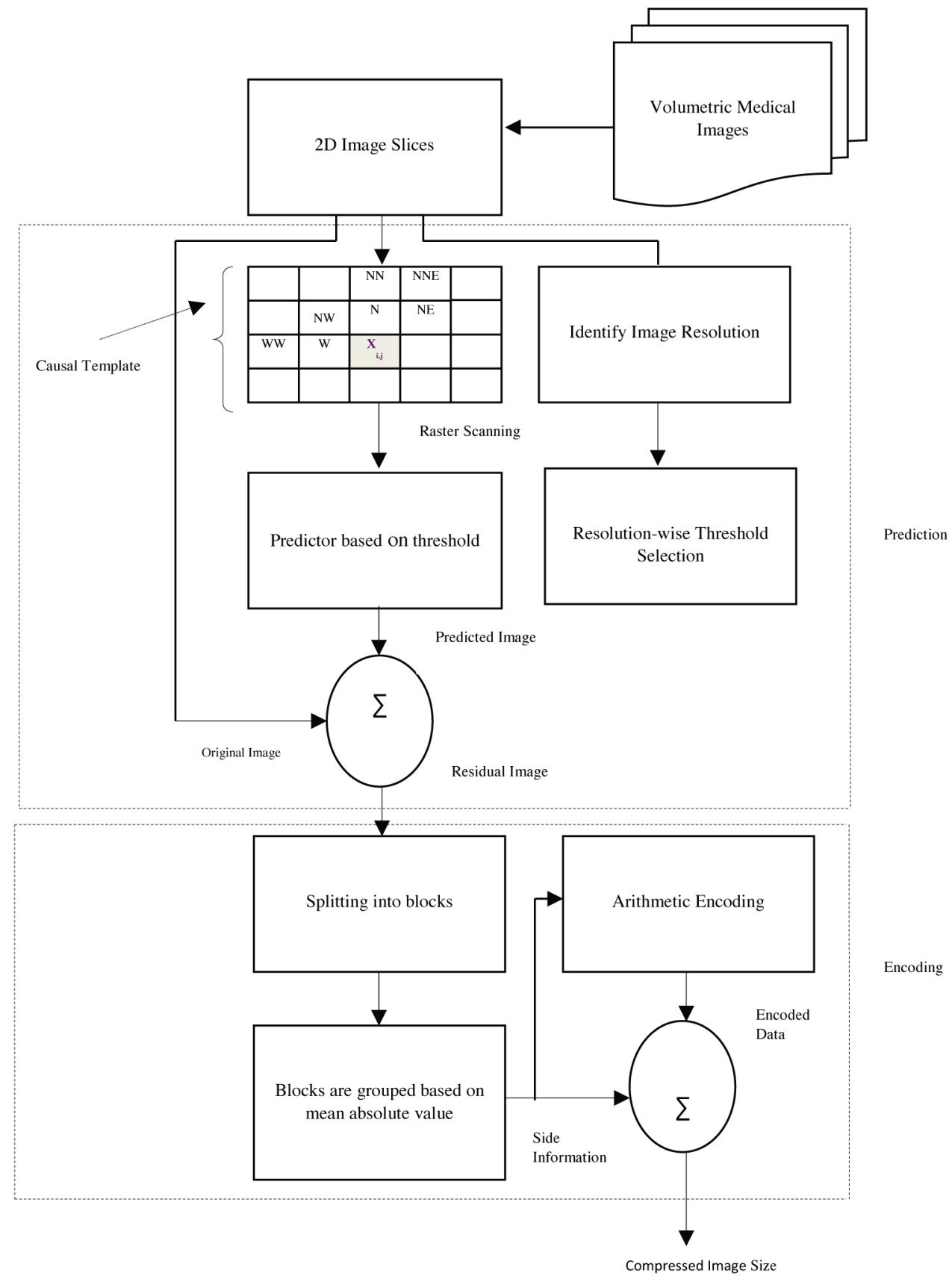

\subsection{Performance Evaluation in RIGED16 Algorithm of Proposed Technique}

In this subsection, proposed algorithm is analyzed for compression performance of volumetric images having different modalities and resolutions. The proposed approach works well for grayscale images of every type of modality. Initially, the coloured images can be converted into greyscale images before prediction. An appropriate threshold value is selected for prediction that can provide the minimum entropy value for all residual images. Our proposed algorithm RIGED16 is designed to make the 
GED algorithm independent of modality and resolution. The GED is tested for different thresholds on the scale of $2^{\text {n }}$. For 16 bit depth images, value of ' $n$ ' can be up to 16 . The entropy values obtained for residual images are predicted at different thresholds and these values are mentioned in Table 3. The weighted average is evaluated for complete dataset on the basis of number of slices.

From results, it is observed that there is a significant variation on entropy value for different threshold levels on the basis of $2^{\mathrm{n}}$. The change in entropy value is quantified as 6.71 to 6.33 when Tlevel ranges from $2^{3}$ to $2^{9}$. Whereas, there is minor variation in the value of entropy when threshold value ranging from $2^{9}$ to $2^{10}$. After $2^{10}$, entropy again starts increasing as shown in Figure 5 . The T-level can be up to $2^{16}$ for 16 bit depth image dataset, entropy value is calculated up to $2^{16}$, but it keeps on increasing after $2^{10}$. Hence, T-level $2^{10}$ can be considered as an optimal value providing minimum entropy.

As the minimum entropy is obtained between the range of T-levels $2^{9}$ to $2^{10}$. Further, the range of T-levels are checked between $2^{9}$ and $2^{10}$ with the difference of 128 (i.e., 640, 768, 896) for optimal universal T-level as shown in Figure 6. There is significant variation in entropy when threshold value changing from 512 to 768 and after 768, entropy again starts increasing. Hence, 768 threshold value is considered as a threshold value that can provide minimum entropy value for 16 bit depth images. For lower and higher thresholds, the entropy value is high but for mid threshold values like 512 to 1024 , entropy value should be minimized. After analysis of threshold value for minimum entropy, it is noted that threshold value (768) provides less entropy value i.e. 6.323.

The weighted average entropy evaluation is done at specific resolution of $256 \times 256$ and $512 \times 512$. The different threshold values are considered to obtain optimal results. The results of this parameter are given in Figure 7. The images with resolution of $256 \times 256$, the minimum value of entropy is obtained at $2^{9}$. For higher resolution images i.e. images of $512 \times 512,2^{10}$ represent minimum entropy. There is slight variation in entropy value from 6.42 to 6.24 when T-levels vary from $2^{9}$ to $2^{10}$ but significant change is observed for higher T-levels i.e. $2^{10}$.

Table 3. Entropy obtained by GED predictor at different threshold values on the basis of $2^{\text {n }}$

\begin{tabular}{|c|c|c|c|c|c|c|c|c|c|c|c|}
\hline \multirow{2}{*}{$\begin{array}{c}\text { Medical } \\
\text { Image } \\
\text { Database }\end{array}$} & \multirow{2}{*}{$\begin{array}{l}\text { Original } \\
\text { Entropy }\end{array}$} & \multicolumn{10}{|c|}{ Entropy Values Obtained at $2^{\text {n }}$ Threshold Levels of 2D GED } \\
\hline & & 8 & 16 & 32 & 48 & 64 & 128 & 256 & 512 & 1024 & 2048 \\
\hline MR_1 & 8.33 & 5.86 & 5.80 & 5.72 & 5.67 & 5.64 & 5.59 & 5.58 & 5.55 & 5.54 & 5.59 \\
\hline MR_2 & 6.99 & 5.09 & 5.06 & 5.02 & 4.99 & 4.98 & 4.95 & 4.94 & 4.93 & 4.92 & 4.95 \\
\hline MR_3 & 8.22 & 6.74 & 6.75 & 6.74 & 6.73 & 6.72 & 6.69 & 6.68 & 6.67 & 6.68 & 6.68 \\
\hline MR_4 & 7.59 & 5.36 & 5.33 & 5.28 & 5.25 & 5.23 & 5.21 & 5.20 & 5.20 & 5.20 & 5.20 \\
\hline MR_5 & 7.63 & 4.80 & 4.73 & 4.64 & 4.59 & 4.56 & 4.48 & 4.46 & 4.44 & 4.43 & 4.46 \\
\hline MR_6 & 7.50 & 6.15 & 6.15 & 6.13 & 6.12 & 6.11 & 6.07 & 6.05 & 6.03 & 6.04 & 6.05 \\
\hline MR_7 & 8.11 & 6.98 & 6.97 & 6.96 & 6.95 & 6.94 & 6.93 & 6.92 & 6.91 & 6.93 & 6.95 \\
\hline $\mathrm{CT}$ & 8.20 & 7.64 & 7.53 & 7.46 & 7.42 & 7.37 & 7.22 & 7.20 & 7.17 & 7.14 & 7.29 \\
\hline $\begin{array}{l}\text { CT_Lung_ } \\
\text { R13 }\end{array}$ & 9.12 & 6.45 & 6.32 & 6.26 & 6.21 & 6.17 & 6.12 & 6.10 & 6.09 & 6.08 & 6.09 \\
\hline $\begin{array}{l}\text { CT-Lung_ } \\
\text { R4 }\end{array}$ & 7.96 & 7.51 & 7.43 & 7.24 & 7.20 & 7.18 & 7.06 & 7.04 & 7.03 & 7.01 & 7.04 \\
\hline MR_Neuro & 7.11 & 6.86 & 6.73 & 6.68 & 6.58 & 6.53 & 6.46 & 6.45 & 6.44 & 6.45 & 6.46 \\
\hline MR_Breast & 8.76 & 7.67 & 7.59 & 7.57 & 7.34 & 7.26 & 7.23 & 7.21 & 7.17 & 7.16 & 7.18 \\
\hline $\begin{array}{l}\text { Weighted } \\
\text { Average }\end{array}$ & 8.49 & 6.71 & 6.62 & 6.54 & 6.48 & 6.45 & 6.39 & 6.36 & 6.33 & 6.32 & 6.36 \\
\hline
\end{tabular}


Figure 5 . Entropy obtained by $2 \mathrm{D}$ predictor at $2^{\mathrm{n}} \mathrm{t}$-levels

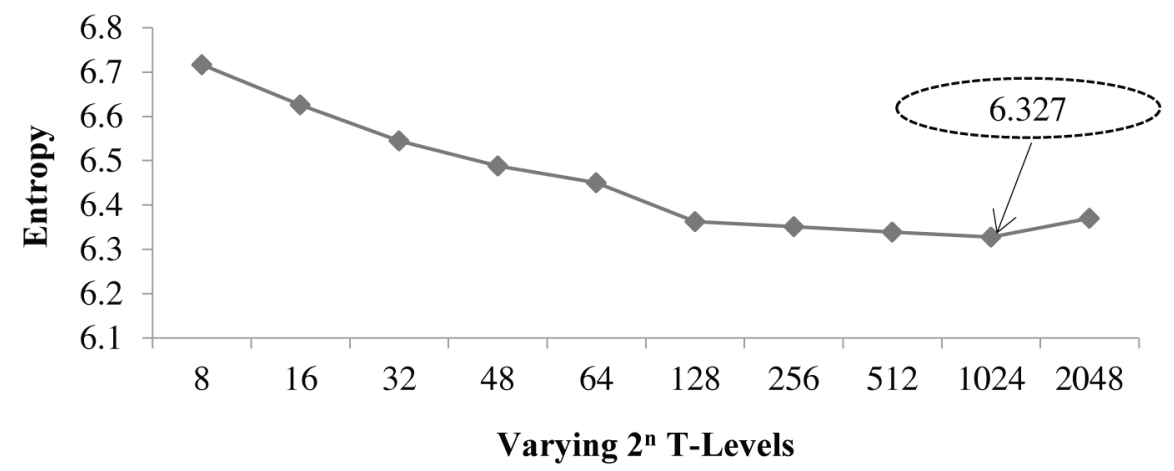

Figure 6. Entropy obtained by 2D predictor at different threshold values in difference of 128

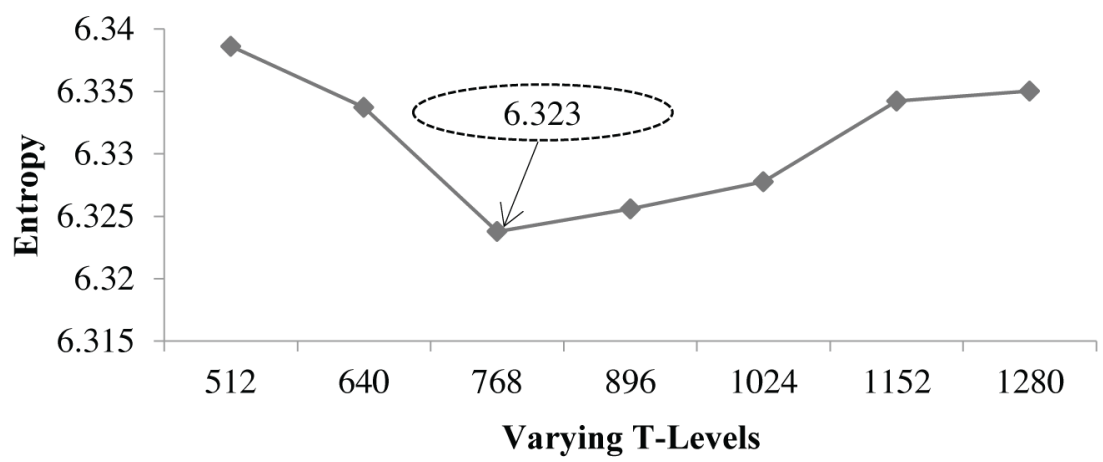

\subsection{Performance Evaluation of BAAE}

The grouping of blocks is done before Arithmetic encoding and BPP values are calculated for different block sizes. The weighted average of BPP is calculated for different block sizes presented in Table 4 . The overhead depends on the block size, BPP values of overhead is also tabulated. It is revealed that the compression performance has a significant variation for different number of block sizes. After experimental analysis on different block sizes, the best block size is selected which finally provides the minimum BPP value. The BPP value with overhead for compressed image is 5.60 at the block size $4 \times 4$. After $4 \times 4$, the BPP value starts decreasing and reaches to 5.07 for a block size of $8 \times 8$. Again, BPP values starts increasing after $8 \times 8$ because overhead decreases with increasing number of block sizes and the code length of compressed image is increases. It is found that when code length of the compressed residual image and overhead information of the block is combined then the lowest BPP value is obtained for a block size of $8 \times 8$. The image datasets include CT-Lung, MR-Neuro, MRBreast and other human parts of different modalities and resolution. But, proposed approach provides same results for BPP. Hence, it is stated that proposed BAAE approach with RIGED16 gives optimal performance in terms of BPP.

A comparison with and without overhead in terms of BPP is shown in Figure 8. It is seen that the minimum value of BPP is obtained for the smallest block size i.e. $4 \times 4$, when BPP overhead is not taken into consideration. The BPP value increases for higher blocks (more than $4 \times 4$ ) due to increase in code-length of residual image. The overhead BPP is maximized for smaller block size i.e. $4 \times 4$, and it decreases with higher number of block sizes (more than $4 \times 4$ ) as shown in Figure 8 . To 
Figure 7. Entropy obtained by GED at $2^{\mathrm{n}}$ threshold values for $256 \times 256$ and $512 \times 512$ resolution

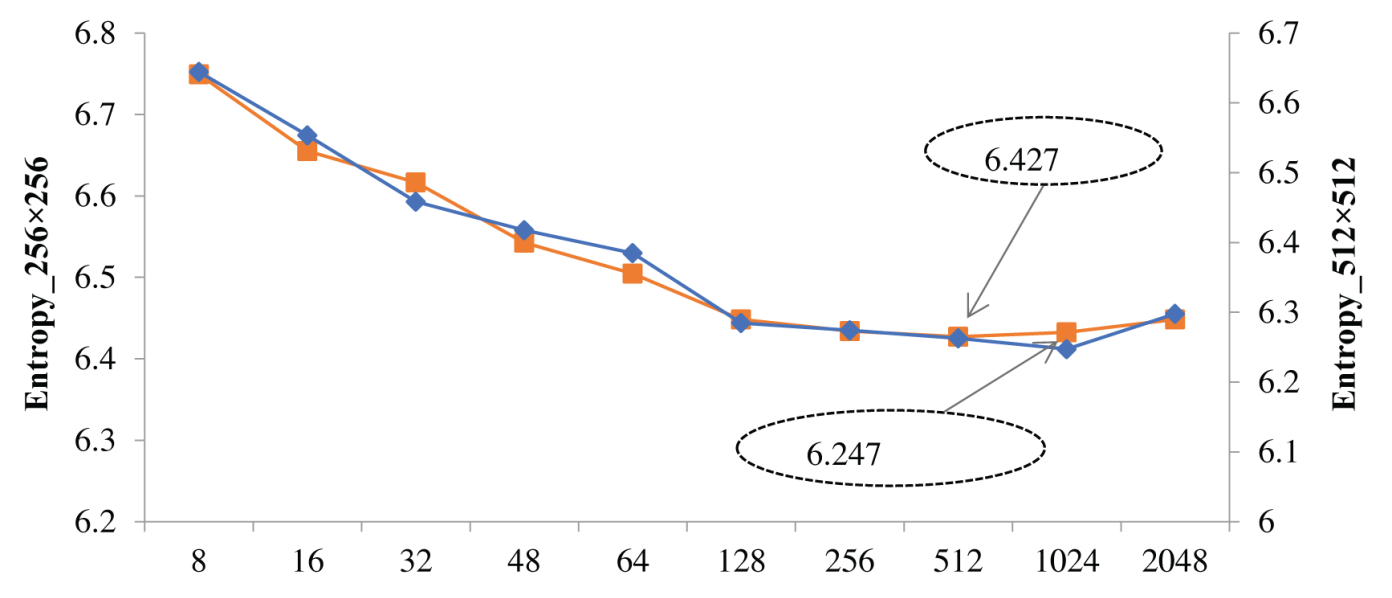

Varying $2^{\text {n }}$ T-Levels

$-256 \times 256$ Resolution $\longrightarrow-512 \times 512$ Resolution

Table 4. Compression results (in BPP) of proposed technique for varying number of block size with overhead

\begin{tabular}{|l|c|c|c|c|c|c|}
\hline \multirow{2}{*}{ Testset } & \multicolumn{5}{|c}{ BPP values for different Block Size (BPP overhead) } \\
\cline { 2 - 7 } & $\mathbf{4 \times 4}$ & $\mathbf{8 \times 8}$ & $\mathbf{1 6 \times 1 6}$ & $\mathbf{3 2 \times 3 2}$ & $\mathbf{6 4 \times 6 4}$ & $\mathbf{1 2 8 \times 1 2 8}$ \\
\hline MR_1 & $4.22(0.2343)$ & $4.08(0.2343)$ & $4.10(0.0548)$ & $4.21(0.0126)$ & $4.36(0.0029)$ & $4.60(0.0006)$ \\
\hline MR_2 & $5.13(0.2343)$ & $5.05(0.2343)$ & $5.06(0.0546)$ & $5.14(0.0126)$ & $5.25(0.0029)$ & $5.40(0.0006)$ \\
\hline MR_3 & $5.97(0.2343)$ & $6.09(0.2343)$ & $6.12(0.0546)$ & $6.27(0.0126)$ & $6.41(0.0029)$ & $6.67(0.0006)$ \\
\hline MR_4 & $4.76(0.2343)$ & $4.68(0.2343)$ & $4.72(0.0546)$ & $4.77(0.0126)$ & $4.86(0.0029)$ & $4.97(0.0006)$ \\
\hline MR_5 & $4.24(0.2343)$ & $4.16(0.2343)$ & $4.18(0.0546)$ & $4.26(0.0126)$ & $4.39(0.0029)$ & $4.54(0.0006)$ \\
\hline MR_6 & $4.90(0.2343)$ & $4.50(0.2343)$ & $4.68(0.0546)$ & $4.76(0.0126)$ & $4.80(0.0029)$ & $4.98(0.0006)$ \\
\hline MR_7 & $4.48(0.2343)$ & $4.46(0.2343)$ & $4.47(0.0546)$ & $4.51(0.0126)$ & $4.54(0.0029)$ & $4.67(0.0006)$ \\
\hline CT & $4.84(0.2343)$ & $4.82(0.2343)$ & $4.94(0.0546)$ & $4.96(0.0126)$ & $4.98(0.0029)$ & $5.39(0.0006)$ \\
\hline $\begin{array}{l}\text { CT_Lung_ } \\
\text { R13 }\end{array}$ & $6.10(0.6837)$ & $5.40(0.1555)$ & $5.42(0.0335)$ & $5.95(0.0074)$ & $6.00(0.0016)$ & $6.05(0.0003)$ \\
\hline $\begin{array}{l}\text { CT-Lung_ } \\
\text { R4 }\end{array}$ & $6.55(0.9411)$ & $5.63(0.2343)$ & $5.63(0.0546)$ & $6.27(0.0126)$ & $6.37(0.0029)$ & $6.55(0.0006)$ \\
\hline MR_Neuro & $5.61(0.5980)$ & $5.08(0.1346)$ & $5.10(0.0300)$ & $5.52(0.0067)$ & $5.75(0.0016)$ & $5.93(0.0003)$ \\
\hline MR_Breast & $4.77(0.9880)$ & $3.90(0.2343)$ & $3.93(0.0546)$ & $4.14(0.0126)$ & $4.36(0.0029)$ & $4.58(0.0006)$ \\
\hline $\begin{array}{l}\text { Weighted } \\
\text { Average }\end{array}$ & $5.60(0.5956)$ & $\mathbf{5 . 0 7}(0.2012)$ & $5.11(0.0461)$ & $5.47(0.0105)$ & $5.57(0.0024)$ & $5.77(0.0005)$ \\
\hline
\end{tabular}

take overhead (including code-length) into consideration, minimum BPP value is obtained for block size $8 \times 8$. The block size more than $8 \times 8$, code length increases and overhead decreases, resulting in minimum value of BPP that can be obtained for the block size $8 \times 8$.

It is clear that there is significant difference between BPP values at $4 \times 4$, with and without overhead. After $8 \times 8$, BPP values obtained with and without overhead are nearly equal and for higher 


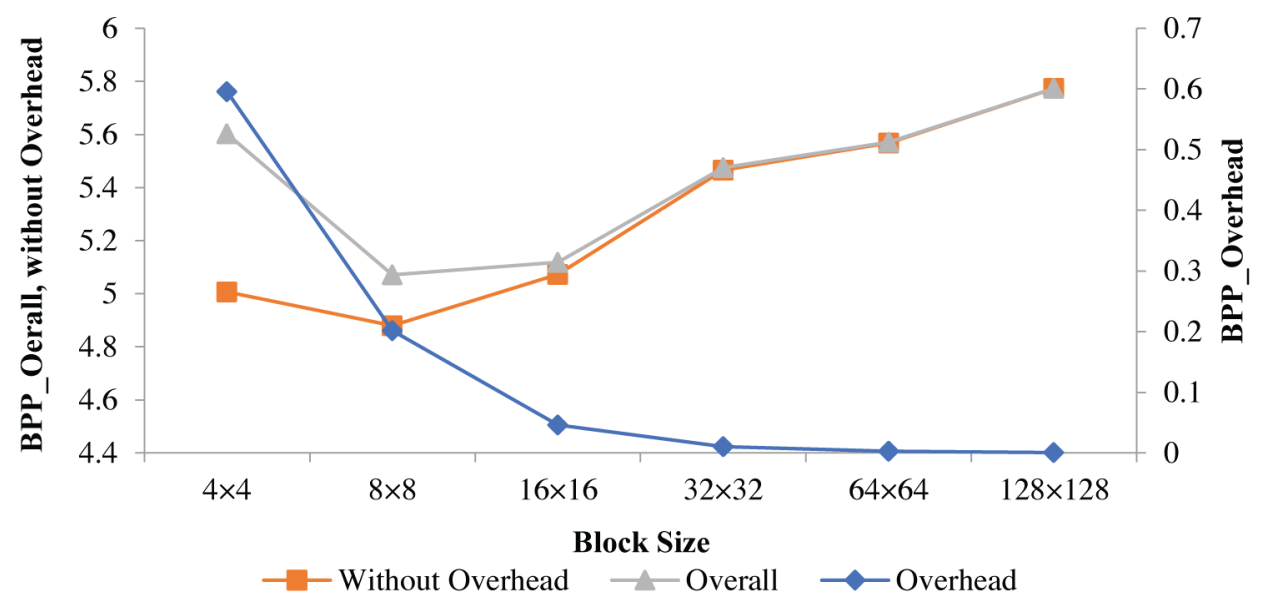

block size after $16 \times 16$, BPP values are equal for both cases, because the overhead is very less resulting in no effect of overhead.

\subsection{Comparison of the Proposed Technique with the Lossless Compression Standards CALIC and JPEG-LS}

Table 5 presents the comparative analysis of compression performance in terms of BPP for the compressed images. BPP values obtained from proposed technique are compared with simple RIGED16 + Arithmetic encoding (without block segmentation). This comparison is made to demonstrate the importance of optimal threshold selection for prediction and the choice of optimal block size for 2D block partitioning of residual images before encoding. From experimentation results, it is observed that the proposed technique performs better in terms of compression ratio compared to RIGED16 + Arithmetic Encoding. The weighted average of BPP using RIGED16 + Arithmetic is 5.93, whereas, the proposed approach obtains BPP values 5.02. When, residual image is directly encoded by Arithmetic encoder (without block partitioning), then a larger value of BPP is obtained as compared to block partitioning image. The proposed approach outperforms than simple RIGED16 + Arithmetic encoding (without blocking) by $17.48 \%$ in terms of BPP values.

Table 6 presents the comparative results of proposed approach and other existing technique like CALIC, JPEG-LS, JPEG-2000. Further, 3D signal, 3D extension of JPEG 2000 (JP3D) (JPEG 2000 codec, 2017), M-CALIC, 3D CALIC (3D-Calic implementation, 2017) and High Efficiency Video Coding (HEVC) (ITU-T and ISO/IEC, 2013) are also used for comparison. It is observed that proposed approach provides more efficient results as compared to other techniques. However, the results are validated by the results obtained on the benchmark dataset.

The proposed RIGED16+BAAE approach achieves 5.29 BPP value, whereas 2D-CALIC and JPEG-LS approaches achieve 5.39 and 5.49 BPP values respectively. The BPP values obtain by other lossless image coding techniques like JPEG 2000, JP3D, M-CALIC, 3D-CALIC and HEVC are 5.55, $5.51,5.75,5.58,5.48$ and 5.36 respectively. Hence, the proposed approach obtains minimum BPP value among all other approaches. The proposed approach is also compared with most recent and highly efficient 3D-MRP algorithm. It is observed that $2 \mathrm{D}$ compression process of proposed approach is simple and efficient and gives comparable results as compared to 3D-MRP-13. The percentage improvement of proposed approach over other lossless coding techniques is given in Table 7.

The proposed method achieves better compression performance and outperforms than standard CALIC and JPEG-LS by $1.90 \%$ and $3.65 \%$ respectively in terms of BPP value. The average 
Table 5. Compression performance results (in BPP) using proposed technique and simple RIGED16+arithmetic encoding for both the test sets given in Section 2

\begin{tabular}{|l|c|c|c|}
\hline \multirow{2}{*}{ Testset } & \multicolumn{2}{|c|}{ Compressed Image Size (BPP) } & $\begin{array}{c}\text { \%Improvement of } \\
\text { Proposed Method }\end{array}$ \\
\cline { 2 - 4 } & $\begin{array}{c}\text { RIGED16+ Arithmetic Encoding } \\
\text { (Without Blocking) }\end{array}$ & $\begin{array}{c}\text { Over } \\
\text { Proposed Approach } \\
\text { EIGED+Arithmetic } \\
\text { Encoding }\end{array}$ \\
\hline MR_1 & 5.04 & 4.08 & 23.45 \\
\hline MR_2 & 5.60 & 5.05 & 10.92 \\
\hline MR_3 & 6.72 & 6.09 & 10.29 \\
\hline MR_4 & 5.22 & 4.68 & 11.60 \\
\hline MR_5 & 4.76 & 4.16 & 14.27 \\
\hline MR_6 & 4.73 & 4.50 & 5.13 \\
\hline MR_7 & 4.79 & 4.46 & 7.21 \\
\hline CT & 5.41 & 4.82 & 3.91 \\
\hline CT_Lung & 6.17 & 5.40 & 14.20 \\
\hline CT-Lung & 6.93 & 5.63 & 23.16 \\
\hline MR_Neuro & 6.36 & 5.08 & 25.31 \\
\hline MR_Breast & 6.62 & 3.90 & 69.53 \\
\hline Weighted Average & $\mathbf{5 . 9 3}$ & $\mathbf{5 . 0 2}$ & $\mathbf{1 7 . 4 8}$ \\
\hline
\end{tabular}

Table 6. Comparison of compression performance (in BPP) using proposed technique and existing compression methods for Test Set I

\begin{tabular}{|l|c|c|c|c|c|c|c|c|c|}
\hline \multirow{2}{*}{ Test-set } & \multicolumn{9}{|c|}{ Compressed Image Size (BPP) } \\
\cline { 2 - 11 } & CALIC & JPEG-LS & $\begin{array}{c}\text { JPEG } \\
\mathbf{2 0 0 0}\end{array}$ & JP3D & M-CALIC & $\begin{array}{c}\text { 3D } \\
\text { CALIC }\end{array}$ & HEVC & 3D-MRP & $\begin{array}{c}\text { Proposed } \\
\text { Approach }\end{array}$ \\
\hline CT_Lung_13 & 5.43 & 5.65 & 5.53 & 5.62 & 6.09 & 5.72 & 5.83 & 5.31 & 5.40 \\
\hline CT_Lung_4 & 5.81 & 5.80 & 6.01 & 5.96 & 5.96 & 5.89 & 5.74 & 5.99 & 5.63 \\
\hline MR_Neuro & 5.11 & 5.29 & 5.36 & 5.16 & 5.38 & 5.34 & 5.06 & 5.04 & 5.08 \\
\hline MR_Breast & 4.11 & 3.93 & 4.16 & 4.07 & 4.28 & 4.22 & 3.94 & 4.00 & 3.90 \\
\hline $\begin{array}{l}\text { Weighted } \\
\text { Average }\end{array}$ & $\mathbf{5 . 3 9}$ & $\mathbf{5 . 4 9}$ & $\mathbf{5 . 5 5}$ & $\mathbf{5 . 5 1}$ & $\mathbf{5 . 7 5}$ & $\mathbf{5 . 5 8}$ & $\mathbf{5 . 4 8}$ & $\mathbf{5 . 3 6}$ & $\mathbf{5 . 2 9}$ \\
\hline
\end{tabular}

compression gain of $4.99 \%$ and $4.18 \%$ is achieved over JPEG 2000 and over its 3D extension (JP3D). On comparing with remaining coding techniques M-CALIC, 3D-CALIC and HEVC, percentage improvement of proposed RIGED16+BAAE technique is $8.64 \%, 5.52 \%$ and $3.47 \%$ respectively. When compared with 3D-MRP then the percentage difference of proposed RIGED16+BAAE and $3 \mathrm{D}-\mathrm{MRP}$ is $1.65 \%$. The improvement in compression performance is due to the selection of optimal threshold prediction value for higher inter-pixel redundancy removal and optimal block size selection for removing coding redundancy in the proposed technique. 
Table 7. Percentage improvement by proposed technique over existing compression methods for Test Set I

\begin{tabular}{|c|c|c|c|c|c|c|c|c|}
\hline \multirow[t]{2}{*}{ Test-set } & \multicolumn{8}{|c|}{ \%Improvement of Proposed Approach } \\
\hline & 2D-CALIC & $\begin{array}{c}\text { JPEG- } \\
\text { LS }\end{array}$ & $\begin{array}{c}\text { JPEG } \\
2000\end{array}$ & JP3D & M-CALIC & $\begin{array}{c}\text { 3D } \\
\text {-CALIC }\end{array}$ & HEVC & 3D-MRP \\
\hline CT_Lung_13 & 0.59 & 4.63 & 2.44 & 4.05 & 12.84 & 6.01 & 8.01 & -1.72 \\
\hline CT_Lung_4 & 3.2 & 2.98 & 6.74 & 5.98 & 6 & 4.7 & 1.98 & 6.37 \\
\hline MR_Neuro & 0.73 & 4.19 & 5.62 & 1.59 & 5.94 & 5.11 & -0.29 & -0.78 \\
\hline MR_Breast & 5.24 & 0.65 & 6.57 & 4.19 & 9.77 & 8.21 & 0.97 & 2.53 \\
\hline $\begin{array}{l}\text { Weighted } \\
\text { Average }\end{array}$ & 1.90 & 3.65 & 4.99 & 4.18 & 8.64 & 5.52 & 3.47 & 1.65 \\
\hline
\end{tabular}

\section{CONCLUSION}

This paper presents a new image compression algorithm based on threshold selection for prediction and choice of optimal block size of residual image for encoding. The proposed approach is developed for efficient lossless compression of volumetric images using RIGED16. Further, block based arithmetic encoding is also used to process volumetric image data independently. The effectiveness of proposed approach is verified using standard test-set and dataset collected from local hospitals. This approach provides higher compression efficiency as compared to standard CALIC and JPEG-LS techniques. The proposed approach achieves percentage improvement of $1.90 \%, 3.65 \%, 3.47 \%$ and $1.65 \%$ over CALIC, JPEG-LS, HEVC and 3D-MRP techniques respectively. 


\section{REFERENCES}

3d-calic implementation. (n.d.). Retrieved from https://prezi.com/j2ichhip4ls7/calic-implementation/

Al-Khafaji, G. (2013). Image compression based on quadtree and polynomial. International Journal of Computers and Applications, 76(3).

Al-Khafaji, G., \& Al-Mahmood, H. (2016). Lossless Image Compression using Adaptive Predictive Coding of Selected Seed Values. International Journal of Computers and Applications, 141(4).

Al-Khafaji, G., \& Ghanim, N. I. (2017). Medical Image Compression using Hybrid Technique of Wavelet Transformation and Seed Selective Predictive Method. International Journal of Engineering Research and Advanced Technology, 3 .

Al-Mahmood, H., \& Al-Rubaye, Z. (2014). Lossless Image Compression based on Predictive Coding and Bit Plane Slicing. International Journal of Computers and Applications, 93(1).

An open-source jpeg 2000 codec written in c. (n.d.). Retrieved from http://www.openjpeg.org/

Anitha, S. (2015). Lossless image compression and decompression using Huffman coding. International Research Journal of Engineering and Technology, 2, 240-247.

Avramović, A., \& Savić, S. (2011). Lossless predictive compression of medical images. Serbian Journal of Electrical Engineering, 8(1), 27-36. doi:10.2298/SJEE1101027A

Avudaiappan, T., Ilam Parithi, T., Balasuramanian, R., \& Sujatha, K. (2017). Performance analysis on lossless image compression techniques for general images. International Journal of Pure and Applied Mathematics, $117(10), 1-5$.

Barboriak, D. (2015). Data From RIDER_NEURO_MRI. The Cancer Imaging Archive.

Bell, T., Cleary, J., \& Witten, I. H. (1990). Text Compression. Englewood Cliffs, NJ: Prentice Hall.

Bhardwaj, C. (2017). Implementation and performance assessment of compressed sensing for images and video signals. Journal of Global Pharma Technology.

Calic implementation. (n.d.). Retrieved from http://www.ece.mcmaster.ca/_xwu/calicexe/

Clark, K., Vendt, B., Smith, K., Freymann, J., Kirby, J., Koppel, P., \& Tarbox, L. (2013). The Cancer Imaging Archive (TCIA): Maintaining and operating a public information repository. Journal of Digital Imaging, 26(6), 1045-1057. doi:10.1007/s10278-013-9622-7 PMID:23884657

Grove, O., Berglund, A. E., Schabath, M. B., Aerts, H. J., Dekker, A., Wang, H., \& Eikman, E. et al. (2015). Quantitative computed tomographic descriptors associate tumor shape complexity and intratumor heterogeneity with prognosis in lung adenocarcinoma. PLoS One, 10(3), e0118261. doi:10.1371/journal.pone.0118261 PMID:25739030

Gupta, V., Rao, A. G., \& Pandey, K. M. (2013). Integer wavelet transform and predictive coding technique for lossless medical image compression. Int. J. Technol. Explor. Learn, 2(4), 143-150.

Information Technology-Digital Compression and Coding of Continuous-Tone Still Images, document ISO/ IEC 10918-1, ITU-T Rec. T.81. (1991).

Information Technology_Lossless and Near-Lossless Compression of Continuous-Tone Still Images ISO/IEC 14495-1, ITU-T Rec. T.87. (1998).

ISO, Int Std, and IEC JTC. Information technology-JPEG 2000 image coding system-Part 1: Core coding system. ISO/IEC 15444-1. (2001).

ITU-T and ISO/IEC JTC1, High efficiency video coding, ITU-T Recommendation H.265 and ISO/IEC 230082. (2013).

Kabir, M. A., \& Mondal, M. (2018). Edge-Based and Prediction-Based Transformations for Lossless Image Compression. Journal of Imaging, 4(5), 64. doi:10.3390/jimaging4050064 
Lucas, L. F., Rodrigues, N. M., da Silva Cruz, L. A., \& de Faria, S. M. (2017). Lossless Compression of Medical Images Using 3-D Predictors. IEEE Transactions on Medical Imaging, 36(11), 2250-2260. doi:10.1109/ TMI.2017.2714640 PMID:28613165

Matsuda, I., Mori, H., \& Itoh, S. (2000). Lossless coding of still images using minimum-rate predictors. In Proceedings of the 2000 International Conference on Image Processing (pp. 132-135). IEEE. doi:10.1109/ ICIP.2000.900912

Me, S. S., Vijayakuymar, V. R., \& Anuja, R. (2012). A survey on various compression methods for medical images. Internationa Journal of Intelligent Systems and Applications, 4(3), 13. doi:10.5815/ijisa.2012.03.02

Meyer, (2015). Data from RIDER_Breast_MRI. The Cancer Imaging Archive. doi:10.7937/K9/TCIA.015. H1SXNUXL

Pennebaker, W. B., \& Mitchell, J. L. (1992). JPEG: Still image data compression standard. Springer Science $\&$ Business Media.

Puthooran, E., Anand, R. S., \& Mukherjee, S. (2013). Lossless compression of medical images using a dual level DPCM with context adaptive switching neural network predictor. International Journal of Computational Intelligence Systems, 6(6), 1082-1093. doi:10.1080/18756891.2013.816059

Ravishankar, S., \& Bresler, Y. (2011, August). Adaptive sampling design for compressed sensing MRI. In Proceedings of the 2011 Annual International Conference of the IEEE Engineering in Medicine and Biology Society (pp. 3751-3755). IEEE.

Taubman, D., \& Marcelin, M. (2001). JPEG2000: Image Compression Fundamentals, Standards and Practice (2nd ed.). Norwell, MA: Kluwer.

Weinberger, M. J., Seroussi, G., \& Sapiro, G. (2000). The LOCO-I lossless image compression algorithm: Principles and standardization into JPEG-LS. IEEE Transactions on Image Processing, 9(8), 1309-1324. doi:10.1109/83.855427 PMID:18262969

Wu, X., \& Memon, N. (1997). Context-based, adaptive, lossless image coding. IEEE Transactions on Communications, 45(4), 437-444. doi:10.1109/26.585919 
Urvashi is a Research Scholar and Teaching Assistant in the Department of Electronics and Communication Engineering at Jaypee University of Information Technology, Waknaghat, H.P, India. She received her B.Tech degree from the HPTU India in the year 2015. She received M. Tech degree from Jaypee University of Information Technology, Waknaghat, H.P, India in the year of 2017. Her research interests include biomedical image processing.

Dr Emjee Puthooran is an Assistant Professor in the Department of Electronics and Communication Engineering at Jaypee University of Information Technology, Waknaghat, H.P. India. He received his B Tech degree from the University of Calicut. He received M Tech and PhD degree from Indian Institute of Technology, Roorkee. He worked as a research fellow at C-DAC (Erstwhile ER \& DCI), Trivandrum and at IIT Roorkee. He also worked as an Assistant Professor at National Institute of Technology, Jalandhar. His research interests include biomedical image processing, digital signal processing, and soft computing techniques. 